\title{
IDEAS-VERSIONES-GENERACIONES A propósito del Plan Montevideo
}

\author{
Eleonora Leicht Arocena \\ Investigadora en el Instituto de Teoría de la Arquitectura y Urbanismo, FADU, UDELAR. \\ Directoras de tesis: Alicia Novick / Adriana Barreiro \\ eleonora.leicht@fadu.edu.uy
}

\section{RESUMEN}

El artículo presenta algunos aspectos de la tesis doctoral en curso titulada Planes y proyectos territoriales en Montevideo, 1985 en adelante. Este ensayo referirá especialmente a tres capítulos pertenecientes al proyecto de tesis: El marco conceptual del POT -IDEAS-, su complejo proceso de elaboración -VERSIONES, y la planificación derivada a la luz de la LOT -GENERACIONES. El corpus de la investigación hace pivot en el Plan de Ordenamiento Territorial de 1998, que consideramos en Uruguay un instrumento fundante de nuestra cultura planificadora contemporánea. Muchas de las fuentes de información son de acceso público, no obstante, hay bases documentales inéditas disponibles, como las versiones anteriores a la finalmente aprobada, rotuladas como el Anteproyecto de 1996 y el Proyecto de 1997 que permiten restituir el zigzagueante proceso de elaboración del POT.

Palabras clave: Planes urbanos, historia de la planificación urbana, Montevideo

\begin{abstract}
The article presents some aspects of the doctoral thesis in progress entitled Plans and territorial projects in Montevideo, 1985 onwards. This essay will refer especially to three chapters belonging to the thesis project: The conceptual framework of the POT -IDEAS-, its complex process of elaboration - VERSIONS-, and the derived planning in light of the LOT -GENERATIONS. The corpus of research makes pivot in the Plan Montevideo of 1998 , which we consider in Uruguay a founding instrument of our contemporary planning culture. Several sources of information have public access, however, there are unpublished documentary bases available, such as the versions prior to the finally approved, labeled as the Anteproyecto of 1996 and the Proyecto of 1997 that allow to restore the zigzagging process of elaboration of the POT.
\end{abstract}

Keywords: Urban plans, history of urban planning, Montevideo 


\section{INTRODUCCIÓN}

Desde 2008, a nivel la temática de la planificación urbana y territorial ha adquirido una importancia creciente enlas agendas académicas y políticas de Uruguay, al punto que adquiere el rol de política pública prioritaria, tal como fuera pautada por la Ley no. 18.308 de Ordenamiento Territorial y Desarrollo Sostenible (LOT).

Sin embargo, la tradición planificadora del territorio y la ciudad no es totalmente novedosa. Sabido es que Montevideo -en consonancia con otras experiencias contemporáneas- contó con un anteproyecto de Plan Regulador en 1930, con un Plan Director en 1956, que fueron considerados como instrumentos de vanguardia a nivel nacional y regional. Treinta años después, con el retorno de la Democracia en 1985, se va gestando una particular coyuntura que culmina en la aprobación del Plan de Ordenamiento Territorial para el Departamento de Montevideo en 1998, que continua vigente hasta la fecha, y sobre el cual se fueron llevando a cabo reformulaciones y actualizaciones. Ese Plan -conocido como Plan Montevideo o POTpresenta las bases de lo que se llama la "planificación derivada", que consiste en formular instrumentos de planificación a diversas escalas, que van desde el proyecto de detalle al plan especial o parcial. Más recientemente y a partir de la aprobación de la LOT, el impulso planificador ha considerado la escala metropolitana e interdepartamental. En la LOT, que responde a una preocupación por un territorio ampliado y sus problemáticas, se puede reconocer en los lineamientos del Plan Montevideo, una importante fuente de inspiración que se pone de manifiesto tanto en la definición de los instrumentos de ordenación a nivel nacional, así como en sus dispositivos, tributarios del urbanismo español. Si bien los primeros planes y las propuestas más recientes se inscriben en contextos históricos y epistemológicos muy diferentes, es posible encontrar vinculaciones y referencias. En relación a estas superposiciones entre nuevos planteos y diversos antecedentes es posible

\footnotetext{
"poner en evidencia cierta solidaridad que se teje entre los proyectos sucesivos que se conciben para un mismo sitio, son experiencias territoriales sedimentadas que dejan sus huellas en los tiempos largos del territorio." (Novick A., pp. 2, 2009)
}

Desde esa problemática, por un lado, la tesis explora el mundo de las ideas, el contexto académico e institucional que hizo posible la concepción del Plan Montevideo, sus actores -hacedores, promotores- sus propuestas y sus referencias -en relación a otros documentos contemporáneos- así como las diversas versiones que se prepararon antes de ser aprobado. En ese contexto, dicho de otro modo, se trata de analizar sus condiciones de posibilidad y el proceso que fue desde las primeras ideas hasta su aprobaciónconsiderando también las herencias, vínculos y rupturas, más ampliamente las superposiciones que establecen con los documentos anteriores y con el sistema planificador, y en particular las relaciones que se establecen con el Plan Director de 1956.

Por otro lado, la tesis se propone examinar el Plan desde su normativa y el amplio espectro de instrumentos de ordenación que contempla incluyendo aquellos proyectos que no llegaron a aprobarse, que si bien fueron aprobados no llegaron a materializarse.

Este ensayo referirá especialmente a tres capítulos pertenecientes al proyecto de tesis: El marco conceptual del POT -IDEAS-, su complejo proceso de elaboración -VERSIONES- y y la planificación derivada a la luz de Ia LOT -GENERACIONES.

\section{EL MÉTODO}




\section{Corpus de la investigación}

El tema de tesis se inscribe en el campo epistemológico de Investigación en Urbanismoํ.

El corpus de la investigación hace pivot en el Plan de Ordenamiento Territorial de 1998, que consideramos un instrumento fundante de nuestra cultura planificadora contemporánea.

Muchas de las fuentes de información son de acceso público, no obstante, hay bases documentales inéditas disponibles, como las versiones anteriores a la finalmente aprobada, rotuladas como el Anteproyecto de 1996 y el Proyecto de 1997 que permiten restituir el zigzagueante proceso de elaboración del POT.

Estos materiales permiten iluminar varias aristas de la problemática a tratar, relaciones entre esfera académica y política, entre tradiciones e innovaciones. Cabe considerar que el anteproyecto del POT fue un producto elaborado en la Academia, se trataba de una innovación sustantiva e inspiró muchos de los conceptos presentes en LOT y en los planes posteriores. Lo mismo puede decirse en el contexto institucional, siendo un logro histórico su aprobación en la Junta Departamental.

\section{Objetivos}

\section{Generales}

-Fortalecer el conocimiento y la promoción de la cultura planificadora

-Identificar las modalidades de pensar, proyectar y operar sobre el espacio construido mediante el estudio de los planes

\section{Particulares}

Diacronías

-Examinar las relaciones, continuidades y solapamientos en los procesos planificadores, tomando como pivot el Plan Montevideo de 1998

Sincronías

-Examinar las vinculaciones entre los discursos y práctica planificadora, entre técnica y política, entre la Academia y las instituciones

Presunciones de partida. La excepción y el olvido como problemas de investigación

"El estado de excepción es hoy la norma" Giorgio Agamben

\section{La excepción}

\footnotetext{
${ }^{1}$ La elección de Montevideo en tanto territorio planificado es producto de mi doble condición de docente investigadora en el Instituto de Teoría de la Arquitectura y Urbanismo (ITU) y de funcionaria en el Departamento de Planificación en la Intendencia de Montevideo (IM), lo que me lleva a poner en debate permanente los vínculos entre teoría y práctica del Urbanismo
} 
El tratado de Agamben (2005) que refiere a cuando el derecho ya no puede garantizar el bien común y se recurre por necesidad a suspenderlo, bien puede aplicarse a las normas urbanísticas. Ejemplos abundan a nivel local y global. La planificación urbana y su efectividad para lograr una ciudad más inclusiva está hoy en entredicho.

Son varios temas problema o inquietudes vinculados a procesos y prácticas de planificación en Montevideo. Al inicio del corriente gobierno departamental (julio 2015) algunos anuncios a medias voces de la supresión del Departamento de Planificación del organigrama de la Intendencia, sacudieron bastante las estanterías. Es que dentro de la Institución cuando se necesitan soluciones a corto plazo algunos visualizan a la planificación como un obstáculo para el hacer.

\section{El olvido}

En otro orden de cosas, se constata una falta de memoria institucional con desorden o ausencia de archivos que respalden la producción planificadora. Aún si un instrumento es aprobado en la Junta Departamental, no hay registro muchas veces de la exposición de motivos de las definiciones urbanísticas para la ciudad, de los procesos y de los participantes. En muchas oportunidades se parte de cero por desconocimiento de la existencia de antecedentes que no fueron cuidadosamente conservados.

\section{La Tesis como aporte a la cultura planificadora}

Montevideo por su condición de capitalidad es la ciudad donde convergen más planes y proyectos urbanos en el Uruguay, ejecutados o no. La Tesis pretende aportar a la puesta en valor de la cultura planificadora, por lo que se la referencia a un linaje de trabajos investigativos que van en la misma dirección.

Existe abundante bibliografía respecto a la historia urbanística y edilicia de la ciudad de Montevideo previa a Ios 70. Al menos dos autores esbozaron una historia urbana por un llamado a concurso del año 1968 para realizarla, promovida por la Junta Departamental. Se trata de Alfredo Castellanos (primer premio) y BarachiniAltezor y (segundo premio), ambos trabajos publicados. El ITU a través de su Director Gómez Gavazzo fue clave en la elaboración del Plan Director de Montevideo de 1956. Posteriormente a 1985, se hicieron en dicho Instituto muchos estudios sobre Montevideo en el marco de la elaboración de instrumentos de ordenación por trabajos en convenio con la Intendencia (Plan para el Cerro, estructurador urbano La Paz-Galicia, POT y su revisión) y con el MTOP, así como publicaciones que estudian la vinculación entre densidades y morfología, la involución urbana, usos del suelo, etc. Además del ITU, el Grupo de Estudios Urbanos (GEU) liderado por Mariano Arana y el Taller de Investigaciones Urbanas y Regionales (TIUR) con Thomas Sprechmann al frente realizaron decisivos aportes al conocimiento de Montevideo. El Instituto de Historia de la FADU aporta en el marco de elaboración del POT un compendio de los planes realizados para la ciudad de Montevideo.

Se identifican por lo menos dos tesis de maestría emparentadas en un linaje que indaga en los procesos urbanos montevideanos. "La ordenación del territorio en áreas rurales. Cambios y permanencias. El caso Montevideo" de Manuel Chabalgoity (2008), e "Hilos Rotos" de Laura Alemán (2012). En esta última se identifica la constante necesidad de acudir a la "lógica del reemplazo" en los procesos de planificación a nivel nacional, lo que implicaría el descarte de un paso anterior.

\section{Las preguntas de investigación}

¿Cuál fue el contexto político y disciplinar que habilitó la concepción del POT? ¿Quiénes fueron sus autores? Que referentes e ideas manejaban? ¿Qué debates conceptuales se dieron durante su concepción? 
¿Cuáles fueron las versiones del Plan antes de la definitiva?

¿Cómo ha sido evaluado a la luz de 20 años de implementación?

¿Qué instrumentos se elaboraron bajo el ala del POT?

¿Cuáles fueron las continuidades con el sistema planificador heredado?

¿Qué hubo de nuevo en el POT?
VERSIONES

GENERACIONES

Algunas de las preguntas tienen su respuesta en el presente artículo. El índice final de la tesis se ordenará de acuerdo a las preguntas planteadas, cada capítulo girará en torno a una interrogante disparadora.

\section{LOS PRODUCTOS}

Como se mencionó en la Introducción, en esta ocasión se expone un avance de los capítulos de la tesis vinculados al marco conceptual del POT, tres cortes analíticos del mismo en su proceso de elaboración, y la planificación derivada a la luz de la LOT.

\section{IDEAS. EL PENSUM DETRÁS DEL PLAN}

Las nociones de Plan y proyecto, son nociones construidas históricamente. El término Plan viene de plano, dispositivo que, al igual que en la arquitectura, permite formalizar el proyecto en una dimensión distinta a la de materialización (Novick, 2009)

La década de los 90, época en que el POT se va concibiendo y madurando hasta su aprobación en 1998, puede identificarse en el plano local, como una etapa de transformaciones en lo disciplinar, donde identifico la coexistencia de dos posturas respecto a la planificación, que pueden leerse no como antagónicas sino complementarias: el "Urbanismo Urbano" (la postura dominante) y el "regreso del Territorio" (la postura emergente). Ambas posturas, se dejan entrever en el POT pero cada una de ellas con alcances bien diferentes.

"El Plan Montevideo, por su parte, encuadra en un contexto teórico internacional en materia de ordenamiento urbano-territorial que toma nuevo impulso en los últimos años, lo cual constituye un desafío, al tratar de condensar la experiencia disciplinar y técnica reelaborada y aplicada a la realidad particular de Montevideo (...)

(...) Sin embargo, en el mundo de las ideas urbanísticas el Plan Montevideo tiene su continuidad. Así, podemos encontrar sus raíces en las iniciativas de los años 80 , en cuanto a reconocer la diversidad histórica de las partes de la ciudad y el valor del patrimonio heredado, en contraposición a las nociones de homogeneidad propias del pensamiento urbanístico moderno, que habían tenido su expresión en las ordenanzas aprobadas en 1947"

POT, pág. 18, I.3.1 Contexto planificador

\section{El Urbanismo Urbano: La postura dominante}

EI Plan Territorial estaba re-emergiendo en el momento de elaboración del POT, por lo que en el mismo se enuncia la necesidad de atender la cuestión metropolitana, y la puesta en valor de las zonas rurales del Departamento, entre otros aspectos. 
Pero en los hechos el Plan en su versión Proyecto (1997) se dedica en buena medida a desarrollar cuestiones relacionadas con el "Urbanismo Urbano", asignando un espacio importante de su documentación escrita y gráfica a la definición morfológica de sus áreas urbanas, estructuradores, áreas caracterizadas, centralidades y equipamientos. Lo metropolitano y su problemática emergente, como el crecimiento de la ciudad informal, si bien mencionado como contexto, no es materia prioritaria a ser abordada por el Plan.

Algunos Talleres de Facultad de Arquitectura estaban alineados con dicha postura, y participaron activamente en el Anteproyecto del Plan unos pocos años después ${ }^{2}$. El equipo académico del Anteproyecto (1996) estaba dirigido por tres arquitectos directores de Taller -Parodi, Folco y Otero- todos, y quizá por ese motivo, acorde con la concepción disciplinar del momento, la dimensión ambiental fue una componente más discreta, aunque no ausente. De hecho en el Proyecto se identifican varias Áreas de Protección Ambiental (Santa Lucía, Costa Oeste, Bañados de Carrasco). La Intendencia reforzaría posteriormente esta mirada con asesoramiento específico del experto en ecología Eduardo Gudynas.

La mirada del Urbanismo Urbano se apoyaba en varios pilares. La obra teórica y proyectual de Aldo Rossi tuvo una fuerte influencia en toda una generación de arquitectos uruguayos, que apostaron a la comprensión estructural de la ciudad por partes. Esta postura se ve reflejada en la reflexión teórica nacional de la década de los 80 y principios de los 90 que se condensa en hemerotecas locales, publicaciones y eventos clave.

Los trabajos realizados por el GEU tuvieron un impacto significativo denunciando la destrucción del patrimonio de la ciudad-especialmente de la Ciudad Vieja- por parte del entonces gobierno dictatorial. Los audiovisuales "Una Ciudad sin Memoria" de 1980 y "¿A quién le importa la ciudad?" de 1983, y por lo menos una docena de publicaciones por parte del GEU entre 1983 y 1987, marcaron una actitud militante en cuanto a cómo resolver la problemática urbana.

En la misma dirección, en 1984, se elabora y presenta el «Informe Áreas Caracterizadas», realizado por convenio entre la Sociedad de Arquitectos del Uruguay y la Intendencia Municipal de Montevideo ${ }^{3}$, redactado entre otros por José Luis Livni, quien diez años después impulsara el exitoso evento "Día del Patrimonio" (Sprechmann, 2006).

También la publicación de "Propuestas a la Ciudad" (TIUR, 1986) en el marco de una efervescente Facultad de Arquitectura postdictadura, tuvo gran difusión, infiltrándose en los mundos de la política urbana montevideana de los 80 y los 90 .

El urbanismo contextualista, que apostaba a los valores de la identidad, el patrimonio y a la construcción de la ciudad por partes, tuvo su fértil campo de aplicación en ciudades europeas como Berlín, que culminó con la experiencia de la IBA, y Barcelona, con los proyectos vinculados a la Villa Olímpica y a sus espacios públicos, ambos referentes obligados para los estudiantes de arquitectura a principios de los 90. En Latinoamérica, en Argentina el correlato sucede con arquitectos contextualistas como Miguel Angel Roca, o

\footnotetext{
${ }^{2}$ No es casual que mis vivencias como estudiante en los cursos de Urbanismo a inicios de los 90 en el por entonces Taller Folco estén vinculadas al estudio de la ciudad consolidada y sus variantes morfológicas

${ }^{3}$ Vale aclarar que la actual Intendencia de Montevideo (IM) se denominaba anteriormente Intendencia Municipal de Montevideo (IMM). El cambio se originó con la creación del tercer nivel de gobiernomunicipios-en 2009.
} 
docentes militantes de la cuestión urbana como Tony Díaz.

La masa crítica formada en torno al "Urbanismo Urbano" a que se hace referencia, vio en el POT la ilusión de poder aplicar y finalmente poner en práctica todo aquel conocimiento de manera integral. De hecho, gran parte del contenido del POT está dedicado a detallar la tipo-morfología que deberían adquirir sus áreas urbanas, con mucho de la impronta de P. Panerai, a consolidar centralidades con "identidad", así como a concebir un sistema de preservación patrimonial. Se realizaron estupendos trabajos bajo este enfoque, como lo fue el análisis tipomorfológico para el Barrio Reus Norte (IMM, 1992. Inédito)

La experiencia hasta entonces desconocida en el ámbito nacional de un gobierno municipal de izquierda, que asume sus responsabilidades a partir de 1990, dio el marco necesario de "voluntad de cambio" y entendió que Montevideo debía sustituir su Plan Director de 1956, el único plan urbanístico global que había tenido la ciudad hasta entonces. Según testimonios registrados del Prof. Hugo Gilmet en la Junta Departamental en 1997, Oriol Bohigas en oportunidad de una visita que realizó a Montevideo, expresó: "Esta Ciudad tiene todo. Con poco ustedes la pueden mejorar".

No obstante, no tardaron en dejarse oír las primeras críticas en cuanto a las limitaciones del Plan, incluso antes de la aprobación. En efecto, Sprechmann (2006) señala un ensayo elaborado en 1992 en co-autoría con el Arq. Capandeguy, titulado "La Costa de Montevideo, una reflexión estratégica" como el primer texto en el plano local que se interroga sobre los límites del paradigma del Urbanismo Urbano.

Por otro lado, el ITU a través de un Convenio con MVOTMA, abordaba por 1995 un estudio exhaustivo del Área Metropolitana, explorando sus delimitaciones, sus actividades productivas y proceso de conformación territorial entre otros temas, que el POT no registra. Las Estrategias Regionales para el área metropolitana (EROTAM) aprobadas unos quince años después, si bien con alto grado de generalidades, salvaron parcialmente este recorte. Posiblemente no estaban en 1998 las condiciones dadas para trascender lo metropolitano del mero discurso teórico.
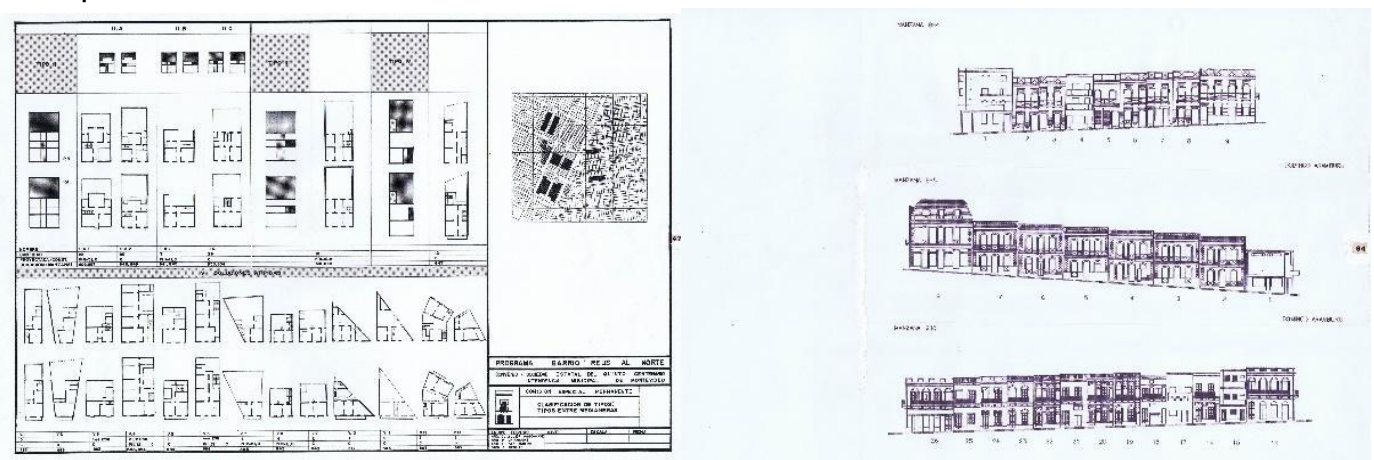


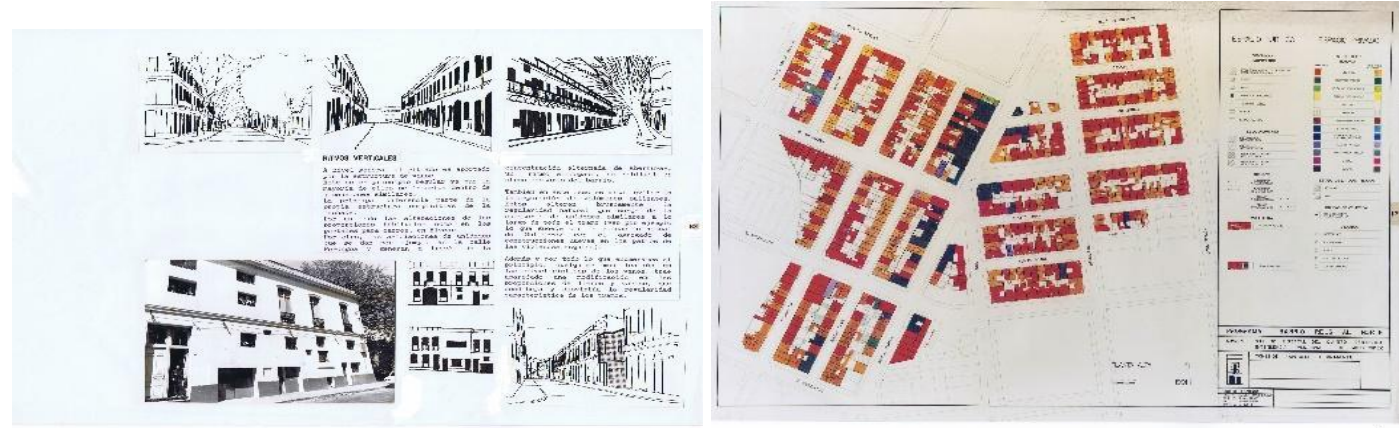

Análisis tipo-morfológicos en Barrio Reus Norte. Fuente: Intendencia Municipal de Montevideo (1992). Programa Barrio Reus Norte. Comisión Especial Permanente de la Ciudad Vieja, Cooperación Española. Inédito

A un año de la aprobación del POT, a modo de temprana evaluación, varios autores coinciden en reclamar el abordaje de lo metropolitano y la atención al crecimiento de la ciudad informal (Ligrone et al, 1999). En 2005 se llevó a cabo un taller de evaluación independiente del POT donde se sugirió un Plan más ágil y se puso en cuestionamiento la idea de la Bahía como rescentramiento de la urbe, ante el inexorable crecimiento de la ciudad costera (CPU arquitectura, 2005. Inédito)

En el contexto español, se puede señalar el artículo de Vegara Gómez de 1993 titulado "Cultura Urbana y Cultura del Territorio", como una temprana caracterización y crítica a los Planes de la Generación de los Ochenta. Se señala en dicho artículo, que si bien estos planes aportaron una nueva Cultura Urbana de gran valor y asociado a la recuperación de los ayuntamientos democráticos, tenían enormes carencias, entre ellas, la negación del hecho metropolitano y de la escala regional, la pretensión de resolver los problemas de la ciudad existente sin atención a posibles demandas futuras, y el rechazo por la utilización de técnicas cuantitativas o instrumentos de base científica en el análisis territorial.

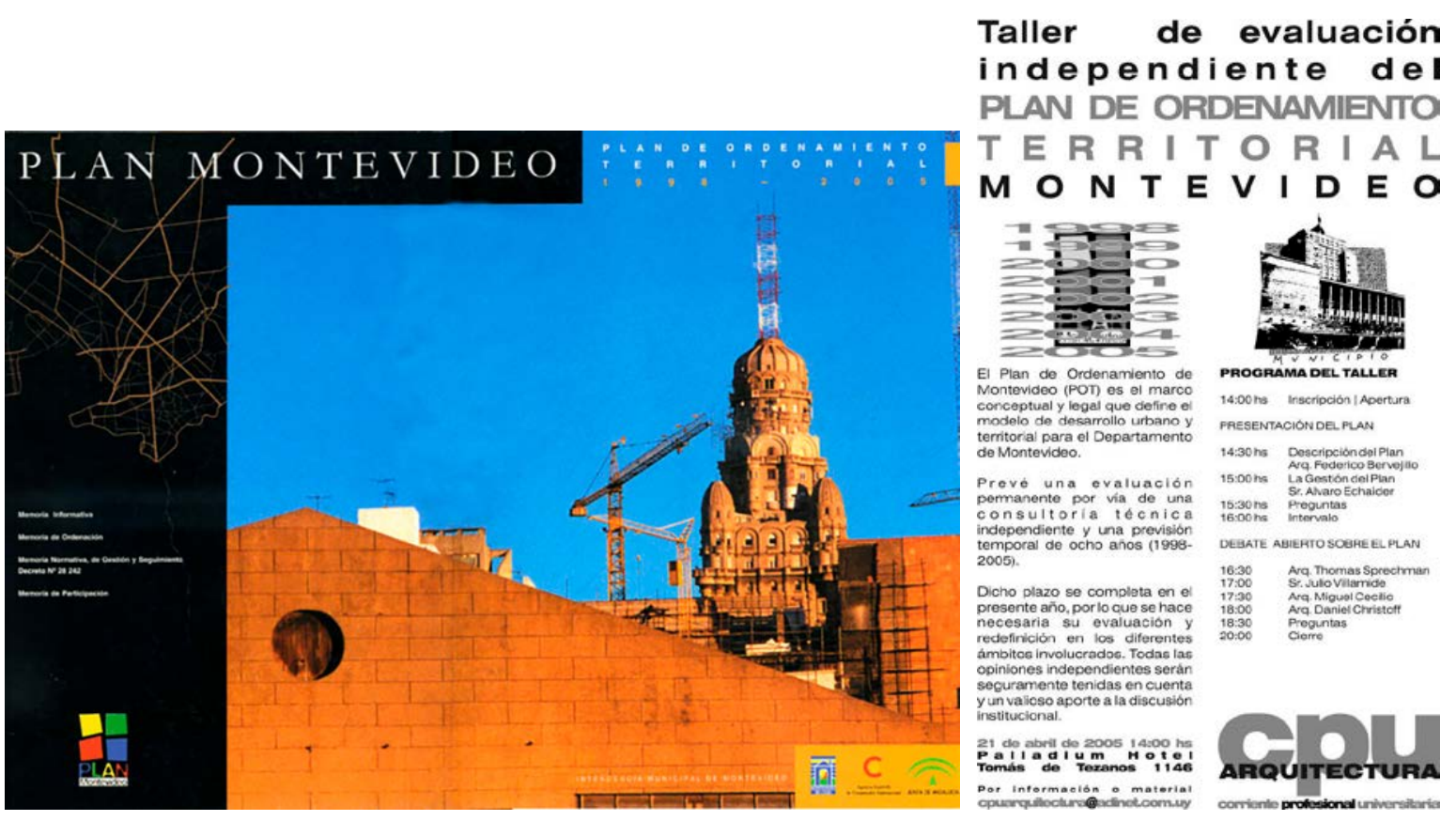


Portada del Plan Montevideo y programa del taller de evaluación independiente

Fuente: Intendencia Municipal de Montevideo (1992) y Corriente Profesional Universitaria (2005). Taller de evaluación independiente del $P O T$. Afiche. Inédito

\section{El Regreso del Territorio: La postura emergente}

Siguiendo con Vegara, este autor identifica los rasgos de la nueva cultura del territorio atendiendo a los retos a futuro para una planificación enmarcada en el siglo XXI. Entre dichos rasgos se subrayan la puesta en valor del medio físico, el tratamiento de las áreas rurales, la conservación de los valores ecológicos, paisajísticos, productivos y culturales. Pone especial énfasis en el tratamiento de las áreas regionales, metropolitanas, así como en las escalas intermedias y escalas locales, y la relación entre ellas. Como instrumentos, menciona como fundamentales el consenso y la ilusión colectiva de la construcción de un modelo territorial y urbano, y la definición de directrices como herramientas idóneas para planificar a la escala regional.

En otro términos, lo que Vegara vislumbra como rasgo emergente no es otra cosa que el retorno de la planificación regional "recargada", en el marco del paradigma de la Sostenibilidad, la Era de la Información y la Sociedad Postindustrial, por citar algunos tópicos que han transformado el Territorio y las miradas al mismo en los últimos treinta años.

En la Era de la Información, con el desarrollo de la tecnología de las comunicaciones y el consecuente colapso de la dimensión espacio/tiempo, como sostienen algunos autores (Castells, Harvey) el enfoque metropolitano adquiere hoy un nuevo significado, mutando la relación entre el individuo y el territorio.

El concepto de territorio está siendo revalorizado en el contexto de la globalización, vinculándose a procesos de desarrollo endógeno y cultura local que se traduce en la idea del "territorio como sujeto" y que implica la recombinación creativa de la magistral vista "desde arriba" con las múltiples miradas "desde abajo". Lo jerárquico se disuelve para dar cabida a lo rizomático.

Respecto al consenso y la ilusión de la construcción colectiva del territorio a que alude Vegara, este concepto está vinculado a una valorización emergente de lo que se ha dado en llamar la negociación entre el Conocimiento Experto (el de los planificadores) y Conocimiento Experimentado (el de los usuarios) en un proceso de aprendizaje mutuo, que ha puesto en relevancia los procesos participativos en la planificación (Friedmann, 1992).

EI POT toma algunos de estos rasgos que identifican la Nueva Cultura del Territorio de manera periférica, no conformando el discurso "dominante". Figuran algunos conceptos renovadores tan sólo como enunciados o exposición de motivos. En la Memoria de Ordenación, si bien el enfoque metropolitano figura como una de las seis ideas fuerzas del Plan, el tema no se desarrolla, quedando en calidad de simple enunciado. Temas que son retomados con más decisión, y ya al amparo de la LOT, en las Directrices Departamentales de 2014 y en las Estrategias Regionales del Área Metropolitana.

\section{TRES VERSIONES SOBRE UN MISMO PLAN}

Se realizan tres cortes analíticos del POT: Anteproyecto, proyecto y versión definitiva. Las fuentes de información de esta sección son directas e inéditas, y permiten dar cuenta del proceso de elaboración del 
POT. Se dejan entrever los puntos de vista convergentes-divergentes entre la Academia y la Administración. Lo que se toma y lo que se deja. Lo que es complejo y se simplifica, o viceversa. Refleja el devenir de un grupo humano heterogéneo puestos a trabajar juntos en un desafío común, en una experiencia sin antecedentes en nuestro medio.

Los documentos inéditos disponibles son los siguientes:

Convenio Intendencia de Montevideo-Facultad de Arquitectura exp.AO 5648, dic. 1995

Carpeta disponible en Intendencia de Montevideo, donde se expresan las condiciones del convenio para la elaboración del Plan, y a que se comprometen las partes. La suma involucrada fueron 585.000 dólares. Figura un listado de la información que aporta la IM, entre ellas el Plan Vial de 1985.

Anteproyecto, diciembre 1996

Documento con textos y cartografía en formato A3 disponible en Intendencia de Montevideo. Posee unas 23 páginas de diagnóstico y mención de antecedentes, y luego gráficos. En la parte propositiva se menciona el anillo colector, y la recalificación de estructuradores cívicos. Entre ellos está propuesto el eje de la Av. 18 de julio desde la Plaza Independencia al Obelisco, con ampliación de veredas, arborización y equipamiento. Se esbozan lo que serían las áreas y proyectos estratégicos; Bahía, Propios, Miguelete, Centro y 18 de julio. Se observan estudios por barrios (Casavalle y Cerro) que se suprimieron en la versión definitiva de 1998.

Gráficos: Propuestas de terminales, luego suprimidas. Diagnóstico (actividades, estructura espacial, integración urbana) y una propuesta para la centralidad de Paso de la Arena. Proyecto para plaza Independencia, acciones propuestas para La llave de la Bahía, detalle para Parque Capurro. Todo esto se suprimió en la versión definitiva

La lectura del anteproyecto deja entrever un documento en elaboración de equipos trabajando de manera fragmentada, donde los niveles de avance e incluso la forma de trabajo es heterogénea. Algunos se inclinan al proyecto arquitectónico, otros intentan ser más sistémicos como es el caso de la propuesta de Centralidades, o el barrio Casavalle.

Proyecto, diciembre 1997

Documento con textos y cartografía en formato A3 disponible en ITU. Se consolidan equipos de trabajo en torno a temas que serán tratados en el Plan definitivo: Áreas rurales protegidas y no protegidas, áreas urbanas centrales costeras intermedias y periféricas, áreas patrimoniales, sistema de espacios públicos y centralidades, estructura vial y transporte colectivo, asentamientos irregulares. Se ofrecen definiciones de parámetros urbanísticos con una riqueza de matices que se simplifican en la versión aprobada: Compatibilidad de usos, grados de saturación de actividades no residenciales, áreas homogéneas y heterogéneas, propuestas de alturas por Zonales. Análisis de las centralidades, propuesta para Goes, Colón, Paso Molino, Unión. Identificación de Áreas de Protección Ambiental. Propuestas de alturas menos conservadora, y más áreas de oportunidad que la versión definitiva. En áreas rurales se identifican varios sectores para promover actividades turísticas. En el índice se anuncian el proyecto 18 de julio, Bahía, Propios, Casavalle, Malvín, y Plan de Transporte. Sin embargo no están desarrollados.

Me detendré en particular en el Modelo Territorial y en el sistema de centralidades para visualizar el devenir que sufrieron en el proceso de generación del Plan.

En cuanto a la evolución de la concepción del Modelo Territorial, un documento publicado previamente a la elaboración del POT figura un croquis de un urbanista francés, J. T.Herbulot, asesor para el Plan de Saneamiento, quien aventuraba una línea diagonal del bienestar humano mínimo en un mapa de Montevideo. Este puede ser considerado el germen de un modelo territorial que evolucionó después a la concepción de la Avenida Propios como zurcidora de una malla social fragmentada. En el anteproyecto de 1996 con los 
primeros avances cartográficos del Plan figura una primera versión del Modelo Territorial, que luego evoluciona al croquis definitivo, publicado y ampliamente conocido. Podemos preguntarnos si las flechas del modelo definitivo intentan coser esa fractura social identificada en el croquis de Herbulot.
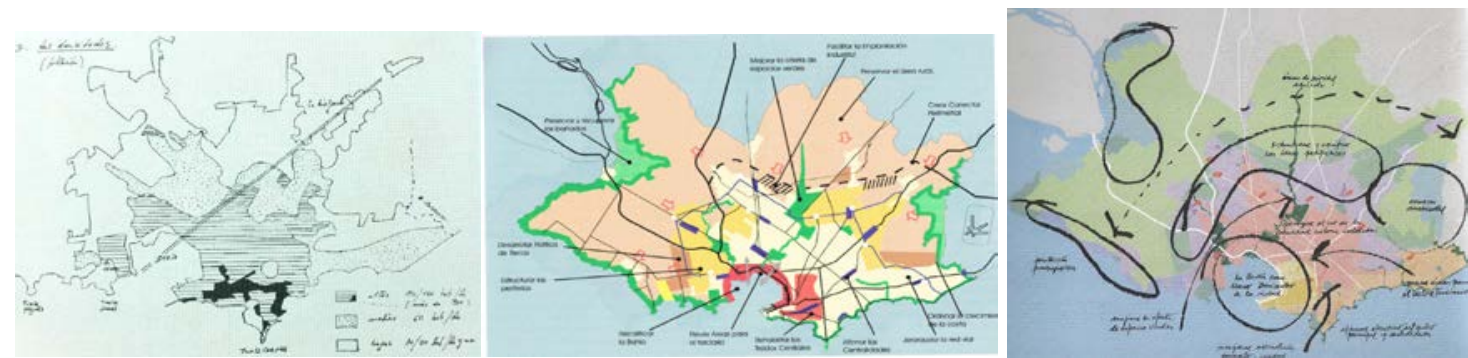

Modelo Territorial del Plan Montevideo. Evolución de su concepción Fuente: 1 J.T.Herbulot. Plan de Saneamiento, IMM, 1992. 2 Modelo territorial en Anteproyecto del POT, Facultad de Arquitectura, 1996. Inédito. 3 Modelo territorial en avance del POT. Hacia un Plan de Montevideo. IMM, 1997

En lo que refiere al tema de Centralidades, la academia realiza un trabajo de detalle exhaustivo de las centralidades urbanas -análisis y propuesta- que luego desaparece en la versión definitiva. El tema preocupaba en ese entonces ya que comenzaban a desarrollarse los shopping centers y se observaban signos de decaimiento de las centralidades tradicionales. Para su análisis, se acudió a datos del Censo Económico que permitía conocer la cantidad de trabajadores en cada comercio, las dinámicas de valores inmobiliarios, etc.

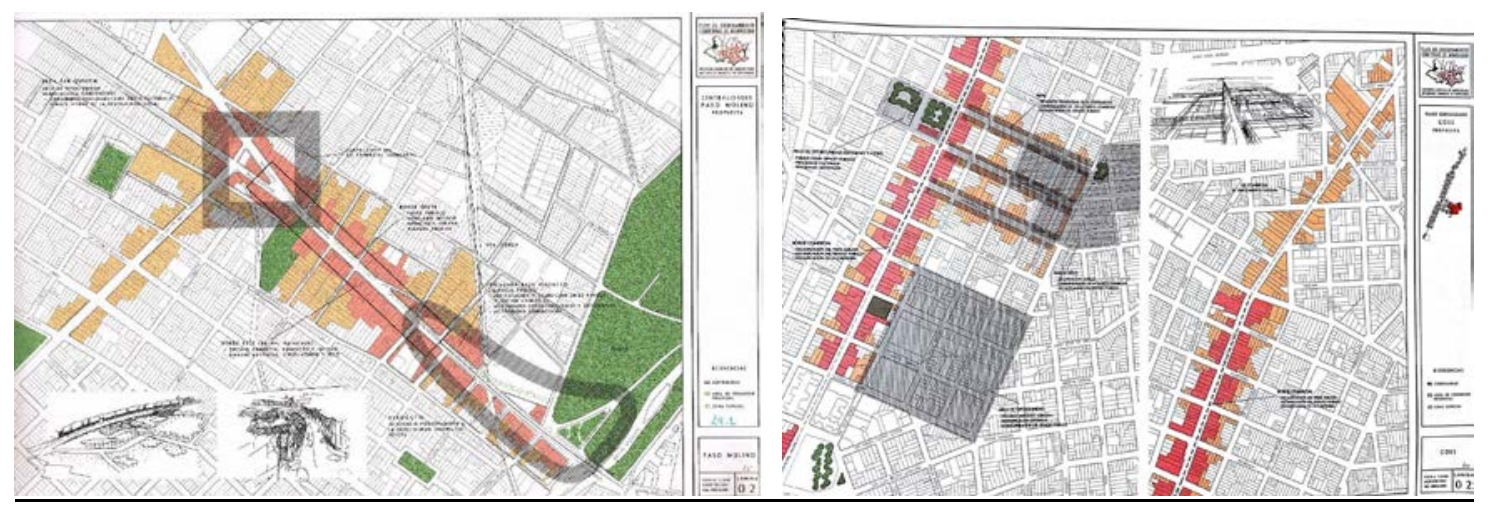

Propuestas para las Centralidades de Paso Molino y de Goes Fuente: Facultad de Arquitectura (1997). Proyecto del Plan de Ordenamiento Territorial. Montevideo, UDELAR. Inédito, disponible en ITU 


\section{GENERACIONES}

En la planificación derivada e Instrumentos complementarios del POT identifico dos etapas en estos veinte años de aplicación del Plan Montevideo, casualmente de idéntica duración. La aprobación de la LOT en 2008 marca un antes y un después en la elaboración de instrumentos derivados y complementarios.
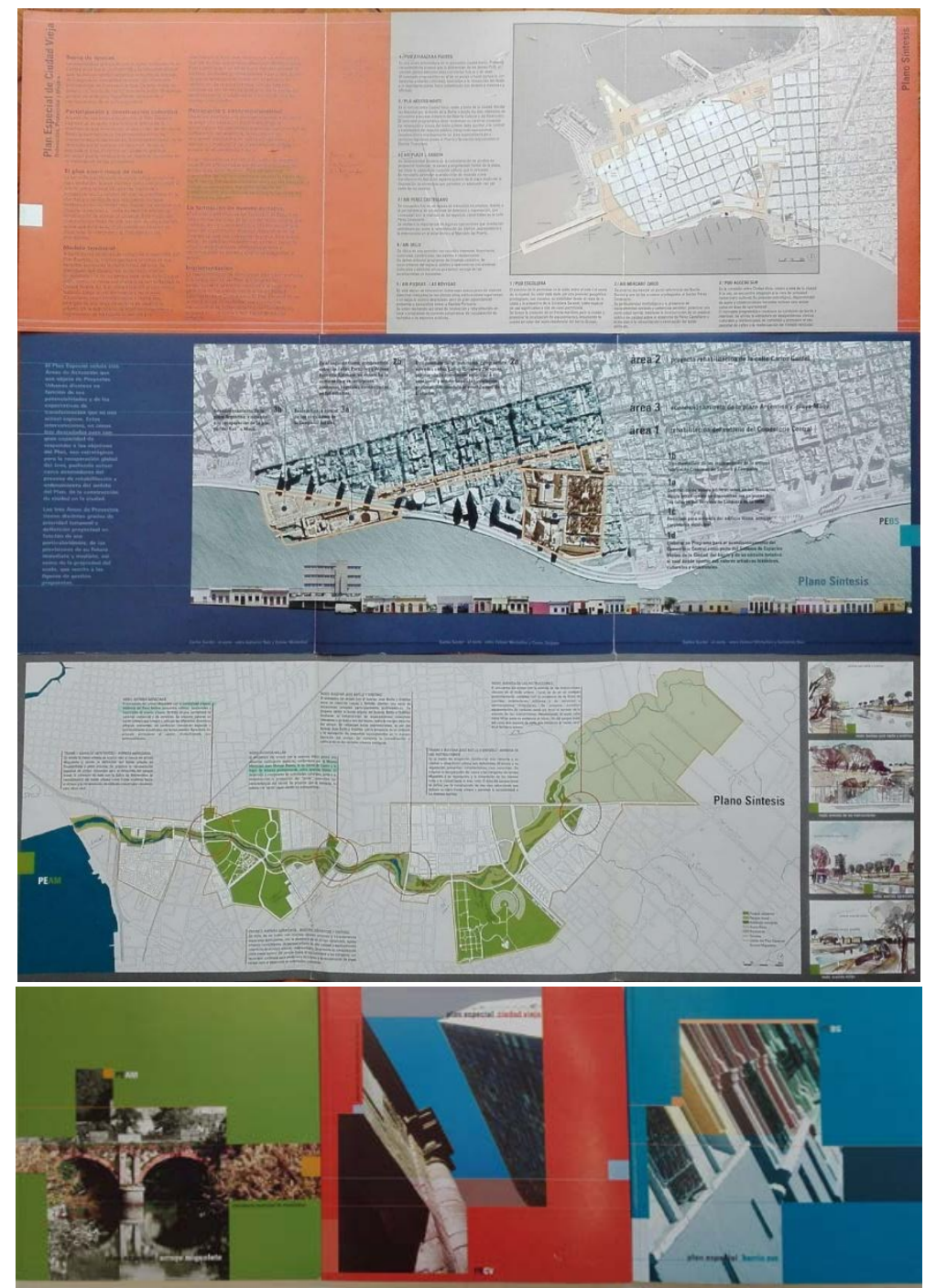

Planificación derivada de primera generación. Barrio Sur, Ciudad Vieja, Miguelete. Planos síntesis y portadas de las publicaciones

Fuente: INTENDENCIA MUNICIPAL DE MONTEVIDEO (2004). Plan Especial Barrio Sur, Plan Especial Ciudad Vieja, Plan Especial Arroyo Miguelete

\section{Los planes de primera generación 1998-2008}

La planificación derivada se pone en marcha atendiendo primeramente planes en áreas patrimoniales. Se 
aprueban en la Junta Departamental el Plan de Ciudad Vieja, Plan del Miguelete y Barrio Sur, así como el PAU 10 (Programa de Actuación Integrada), al norte de Carrasco, atendiendo a demandas de transformación de suelo rural a urbano tipo barrio jardín con fines residenciales para sectores de ingresos medios y altos. El Plan de Ciudad Vieja que incluye en su ámbito la Avenida 18 de julio hasta Ejido fue pionero en la elaboración de un inventario patrimonial con asignación de grados de protección a todo el padronario, y a los tramos de calle, integrando el espacio público. Se llega al detalle de valorizar vistas del paisaje urbano y se proponen cuatro proyectos de detalle a priorizar. Fue objeto de consultoría previa con un equipo externo a la Intendencia, y contó con el asesoramiento del Instituto de Historia para la realización de dicho inventario.

Esta etapa se caracteriza por una confianza renovada en la cultura del Plan que se traduce entre otras cosas por la publicación de una "colección de planes" para difusión a la comunidad. Se contó en la mayoría de las veces con el apoyo económico de la Cooperación Española.

Plan Especial de Barrio Sur: Se activan los proyectos de detalle 10 años después. Por mucho tiempo el Plan Especial de Barrio Sur, aprobado en 2004 en plena crisis económica, parecía uno de los tantos instrumentos destinados a permanecer solo en el papel, con excepciones de mejoras en torno a la calle Carlos Gardel y reciclaje de algunas viviendas. Pasados más de diez años de su aprobación, el Plan finalmente concreta sus Proyectos de Detalle, los que traen aparejado mayor impacto poblacional. El proyecto de detalle La Buena Estrella -localizado en la antigua fábrica de jabones Strauch enfrente al Cementerio Central - da lugar a 338 viviendas agrupadas en dos torres amparadas en la Ley de Vivienda de Interés Social. La dinámica de construcción de cooperativas en las manzanas donde se encontraban los galpones pertenecientes al Servicio de Limpieza de la Intendencia-proyecto de detalle Talleres se compara con el impacto densificador del proyecto Entorno de Mercado Agrícola ya mencionado, donde seguramente el proyecto atractor en este caso es la Rambla Sur, a la vez que próximo al Centro y en un sector de singular patrimonio cultural. Entre las flamantes cooperativas de cuatro o cinco niveles, con tipología de manzana de borde cerrado, anchas veredas con incipiente arbolado nos conducen desde Gonzalo Ramírez a las torres en el verde. La demolición del $\mathrm{CH} 20$ de INVE por riesgo de derrumbe abrió un nuevo balcón al mar para disfrute de antiguos y recientes habitantes del barrio. Un sector antes difícilmente accesible hoy se ha convertido en espacio público privilegiado. Y se empiezan a esbozar, entre el movimiento de las obras y las mudanzas, nuevas dinámicas en la zona: más niños y mascotas, más deportistas, también más autos.
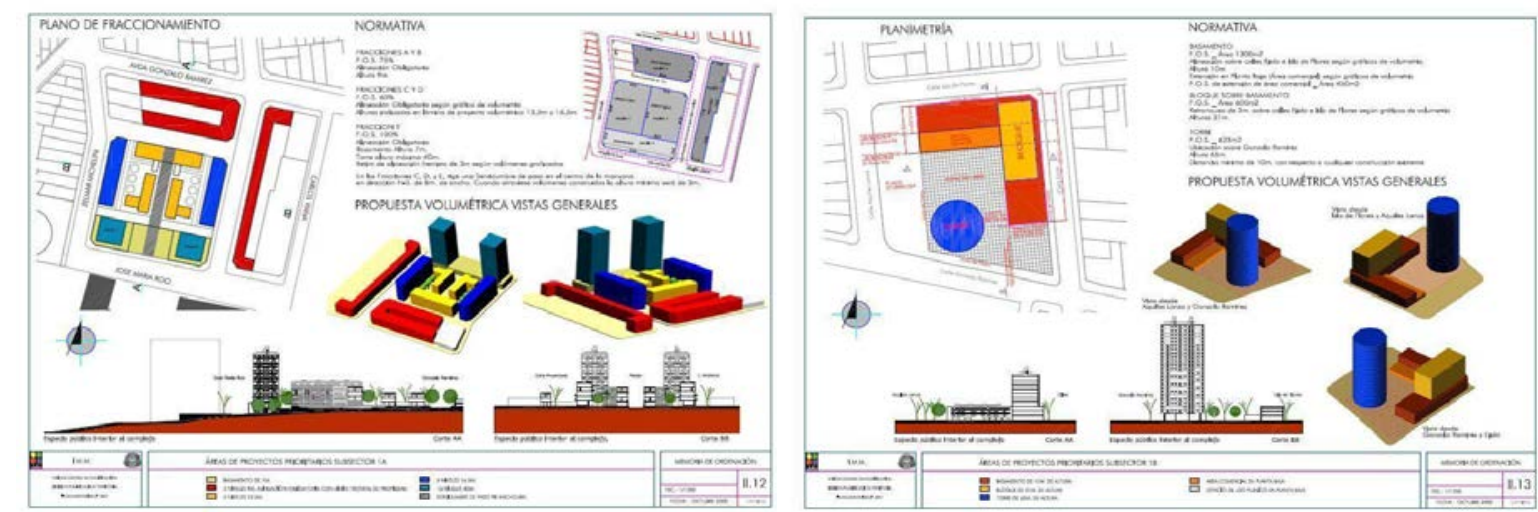

Proyectos de Detalle del Plan Especial Barrio Sur, hoy en construcción. Fuente: INTENDENCIA MUNICIPAL DE MONTEVIDEO (2004). Plan Especial Barrio Sur

Los planes de segunda generación 2008-2018 
Desde la aprobación en 2008 de la LOT, la sostenibilidad ha adquirido un protagonismo aún más decisivo en la formulación de los instrumentos de planificación. Esto resulta evidente en la delimitación de la categorización del suelo y en la definición de los territorios y proyectos estratégicos de las Directrices Departamentales (2014). Las mismas condensan la preocupación por la preservación de los recursos ambientales, la integración socio-territorial y el control de la expansión urbana. Los planes priorizados en el último tiempo son en sectores de cuencas de cursos de agua y bordes de interfase urbano-rural, donde coexiste la problemática ambiental y la presencia de asentamientos irregulares. El Plan Casavalle, Cuenca Chacarita, Cuenca Pantanoso, Arco del Este y Santiago Vázquez y su microrregión son todos instrumentos que dan cuenta de este enfoque.

Esta mirada renovada no impidió que se continuara con instrumentos pendientes en áreas patrimoniales, aprobándose en esta etapa el Plan de Carrasco, Prado, Goes y en proceso de culminación el Plan de Pocitos. Esta etapa se caracteriza por el trabajo interdisciplinario e integrado, y por un reforzamiento de los planteles técnicos de la Intendencia en temas de planificación mediante llamados abiertos de oposición y méritos, después de muchos años sin ser realizados, por una disposición gubernamental que lo impedía. Si bien no se prescinde de las consultorías externas ni de la academia para la elaboración de los instrumentos, la contraparte municipal se encuentra en una posición más saludable para la interacción, con técnicos que por lo general también están en la academia. También los procesos participativos, se inician en etapas tempranas de la elaboración de planes, trascendiendo las instancias exigidas por la Ley, tal como la Puesta de manifiesto y la audiencia Pública. La ley de descentralización en 2009 por la que se crean los municipios abrió la participación a los actores locales, y hoy los instrumentos se realizan en continua interacción con el tercer nivel de gobierno.

La Intendencia de Montevideo fue reconocida por parte del MVOTMA con el otorgamiento de dos de los premios nacionales de urbanismo en su edición 2015, ambos a instrumentos de planificación parcial y de detalle respectivamente.

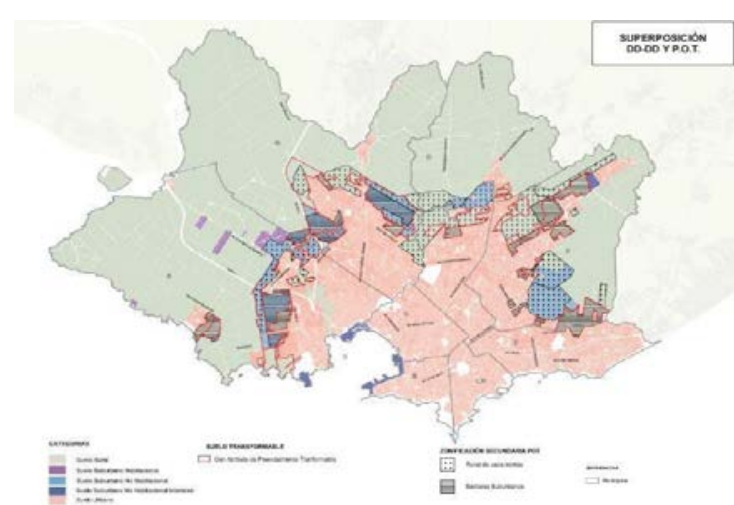

La retracción del suelo urbano y los proyectos estratégicos en las Directrices Departamentales

Fuente: INTENDENCIA DE MONTEVIDEO (2014). Directrices Departamentales de Ordenamiento Territorial y Desarrollo Sostenible

Las Directrices Departamentales y la retracción de lo urbano. Las Directrices Departamentales de Montevideo aprobadas en 2014 replantean la categorización del suelo definida en el POT, combinando un poco de ciudad deseada y ciudad real, y ateniéndose a disposiciones de la LOT. Como bien decía el Profesor Dr. José María Ezquiaga en el VI Seminario Internacional de Investigación en Urbanismo (Bogotá, 2014), América Latina es un campo de fuerzas donde pujan dos tendencias en cuanto al enfoque de los asuntos urbanos: Por un lado la escuela norteamericana donde se tiende a satisfacer la demanda -las cosas son como son-, por otro la escuela europea donde se trabaja desde la oferta, en otras palabras, desde la Utopía o desde la ciudad ideal. Esta idea se hace fácilmente comprensible cuando lo aplicamos a temas concretos como el uso y la 
categorización del suelo. Montevideo a la hora de definir sus directrices obviamente no parte de cero sino que capitaliza la cultura de planificación heredada. No puede más que asumir algunas transformaciones que extienden la mancha urbana, producto de asentamientos irregulares y áreas dedicadas a logística y otras actividades no propias de lo rural. La retracción de lo urbano pasa fundamentalmente por reconocer las cuñas verdes aún rescatables de las márgenes del arroyo Miguelete y Pantanoso, tema fundamental en la agenda ambiental del departamento. Las cuñas verdes - proyectos territoriales de valor estratégico para las directrices- se conciben como paisajes de alta naturalidad asociada a los cursos de agua que ingresan a la ciudad y en consecuencia se les asigna la categorización de suelo rural cuando antes, muchos de esos suelos eran rural de usos mixtos de acuerdo a las normas complementarias, es decir, admitían actividad industrial. Inclusive, atendiendo a observaciones surgidas en los procesos participativos previstos en la LOT durante la elaboración de las Directrices, vecinos de la Costa Oeste logran que disminuya el área transformable a Suelo Urbano del Programa de Actuación Integrada localizado al Este de Pajas Blancas (antes PAU 1). En otras palabras, se recuperó una porción de suelo rural costero.

Plan Parcial de Goes: El entorno del Mercado Agrícola recibe más de 3000 habitantes. Es una de las áreas de intervención prioritaria identificada en el Plan Parcial de Goes en tanto motor y dinamizador de la zona, proyecto clave para lograr el objetivo de revertir el proceso de vaciamiento poblacional, recalificar el espacio urbano y recuperar el stock edilicio. Es un área de intervención donde confluyen distintas políticas, programas y proyectos, donde se integra habitación, espacio público, y el Mercado como gran equipamiento y proyecto estrella. En el entorno del Mercado Agrícola convergen articuladamente diversas intervenciones públicas y privadas que promueven la revitalización de la zona:

-Un mercado en estructura de hierro, antes centro de llegada de productos del cinturón agrícola de la ciudad y por un tiempo prácticamente en desuso, hoy con su recuperación y reapertura se convierte en el corazón de un sector de Goes que se está renovando.

-Un nuevo espacio público como es la Plaza Pepe d' Elía, recientemente inaugurada, y otros espacios proyectados, como la Plaza del Mercado, la que procura otorgar visibilidad al Mercado desde la Avenida General Flores.

-Un importante stock de vivienda de interés social, que adquiere el impacto poblacional que al inicio del siglo XX tuviera el sector de valor patrimonial Reus Norte, dando acogida a más de 3.000 nuevos habitantes.
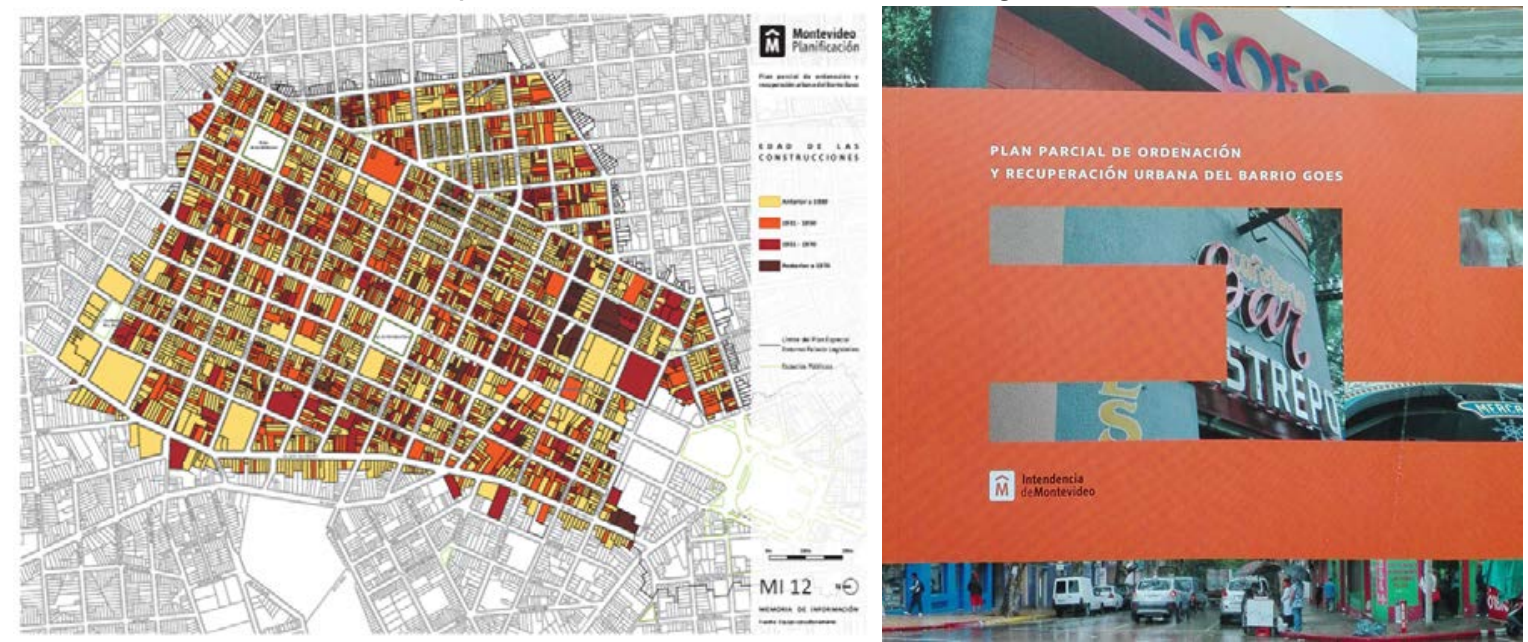

Planificación derivada de segunda generación. Goes. Plano de diagnóstico y portada de la publicación

Fuente: INTENDENCIA DE MONTEVIDEO (2014). Plan Parcial de Ordenación y Recuperación Urbana del Barrio Goes 


\section{CONCLUSIONES}

El artículo brinda un panorama de la cultura planificadora en el territorio montevideano en el marco de la aprobación del POT, poniendo en evidencia en esta ocasión los aspectos conceptuales, el proceso de generación del instrumento, los avances de la planificación derivada y el grado de concreción de alguna de sus propuestas. La información de base fue obtenida de referencias bibliográficas y acceso a fuentes directas de información inéditas. Constituye un avance de algunos aspectos de la tesis doctoral en curso, queda pendiente desarrollar entrevistas a actores clave que echarán luz al tema de investigación con su testimonio y permitirán el desarrollo de algunos capítulos pendientes: los referentes extranjeros y el debate local, el equipo de trabajo y la cooperación española, las microhistorias en torno al Plan.

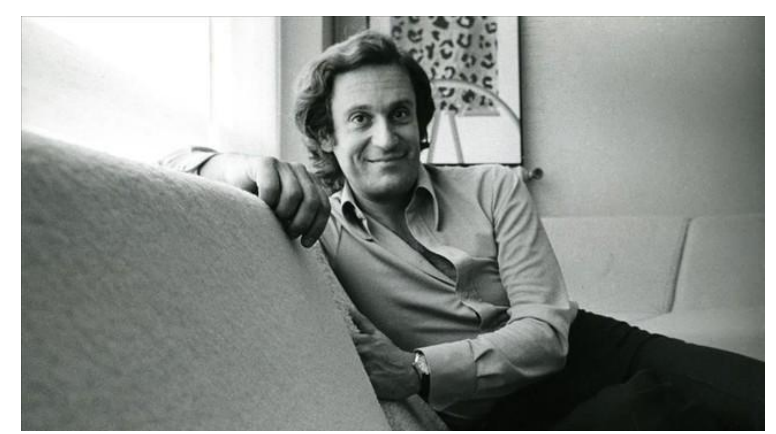

El mensaje optimista de Oriol Bohigas, a propósito de Montevideo en 1997: "Esta Ciudad tiene todo. Con poco ustedes la pueden mejorar" Fuente: Junta Departamental de Montevideo (1996-1997). Actas de sesiones: presentaciones de avances del POT 


\section{BIBLIOGRAFÍA}

AGAMBEN, GIORGIO (2005). Estado de excepción. Buenos Aires. Adriana Hidalgo Editora

ALEMÁN, LAURA (2012). Hilos rotos. Ideas de Ciudad en el Uruguay del siglo XX. Montevideo, Editorial HUM BOISIER, SERGIO (2005). Curso de Epistemología y Axiología territorial. Maestría en Ordenamiento Territorial y Desarrollo Urbano. Montevideo. Multicopiado

CARMONA, LILIANA Y GOMEZ, MA. JULIA (1999). Montevideo. Proceso planificador y crecimiento. Montevideo. FAU-SAU

EZQUIAGA, JOSÉ MARÍA (2014) Conferencia de apertura en el VI Seminario Internacional de Urbanismo, Bogotá https://upcommons.upc.edu/handle/2099/15197

CHABALGOITY, MANUEL (2008). La ordenación del territorio en áreas rurales. Cambios y permanencias. El caso Montevideo.[ https://www.colibri.udelar.edu.uy/jspui/handle/123456789/8076[

DEPARTAMENTO DE URBANÍSTICA Y ORDENACIÓN DEL TERRITORIO, ETSA, UPM (2000): "Planeamiento en el Siglo XXI", Urban, nos. 4 y 5

FRIEDMANN, JHON (1992). Planificación para el Siglo XXI. El Desafío del Posmodernismo. Revista EURE No. 55, página 88

GIORDANO, FERNANDO ET AL (2014). Montevideo y el Grupo de Estudios Urbanos. La reivindicación de la ciudad. Montevideo: FADU Ediciones MCV

INTENDENCIA MUNICIPAL DE MONTEVIDEO (1998). Plan de Ordenamiento Territorial

INTENDENCIA MUNICIPAL DE MONTEVIDEO (2004). Plan Especial Barrio Sur

INTENDENCIA MUNICIPAL DE MONTEVIDEO (2004). Plan Especial Ciudad Vieja

INTENDENCIA MUNICIPAL DE MONTEVIDEO (2004). Plan Especial Arroyo Miguelete

INTENDENCIA DE MONTEVIDEO (2014). Plan Parcial de Ordenación y Recuperación Urbana del Barrio

Goes

INTENDENCIA DE MONTEVIDEO (2014). Directrices Departamentales de Ordenamiento Territorial y Desarrollo Sostenible

LEICHT, ELEONORA (1999). Tesis de Maestría: Urban Growth Management for Metropolitan Montevideo.

UK, Oxford Brookes University, Joint Centre for Urban Design

LEICHT, ELEONORA (2011). Seis Desafíos para una actualización del Plan Montevideo. En: Foro Prospectiva Montevideo 2025, CSIC, biblioteca plural. Udelar

NOVICK, ALICIA (2010). Los proyectos territoriales en perspectiva. Planes Proyectos e Ideas para el AMBA.

Buenos Aires: SCA

NOVICK, ALICIA (2011). Proyectos urbanos y otras historias. Buenos Aires: SCA

SPRECHMANN, THOMAS (2006): "Prólogo a la Ciudad Celeste y otros escritos", La Ciudad Celeste (un nuevo territorio para el Uruguay del siglo XXI), Facultad de Arquitectura, Universidad de la República, Fundación Colonia del Sacramento, Montevideo, pp. 8-19

VEGARA GÓMEZ, ALFONSO (1993): "Cultura urbana y cultura del territorio. Los retos del urbanismo del siglo XXI", Ciudad y Territorio. Estudios Territoriales, Volumen I tercera Época no.95-96 primavera- verano

\section{Leyes y Decretos}

Junta Departamental de Montevideo (16-9-1998). Decreto No 28.242. Plan de Ordenamiento Territorial Junta Departamental de Montevideo (5-5-2003). Decreto No 30.317. Plan Especial Barrio Sur Junta Departamental de Montevideo (29-5-2003). Decreto No 30.302. Plan Especial Arroyo Miguelete Junta Departamental de Montevideo. (4-12-2003). Decreto No 30.565. Plan Especial Ciudad Vieja Junta Departamental de Montevideo (4-9-2014). Decreto No. 35.228. Plan Parcial de Ordenación y 
Recuperación Urbana del Barrio Goes

Junta Departamental de Montevideo (14-11-2013). Decreto No. 34870 y modif. Directrices Departamentales de Ordenamiento Territorial y Desarrollo Sostenible

Junta Departamental de Montevideo (1996-1997). Actas de sesiones: presentaciones de avances del POT Poder Legislativo (18-6-2008). Ley No.18.308 de Ordenamiento Territorial y Desarrollo Sostenible

Poder Legislativo (18-9-2014). Ley No. 19.272 de Descentralización y Participación Ciudadana

\section{Documentos inéditos}

Corriente Profesional Universitaria (2005). Taller de evaluación independiente del POT. Afiche y versiones desgrabadas de las ponencias de Thomas Sprechmann y Julio Villamide. Gentileza de Leonardo Altmann Facultad de Arquitectura (1996). Anteproyecto del Plan de Ordenamiento Territorial. Montevideo, UDELAR. Disponible en IM

Facultad de Arquitectura (1997). Proyecto del Plan de Ordenamiento Territorial. Montevideo, UDELAR. Disponible en ITU

Intendencia Municipal de Montevideo (1992). Programa Barrio Reus Norte. Comisión Especial Permanente de la Ciudad Vieja, Cooperación Española

Intendencia de Montevideo (1996). Convenio Ao. 5648 i.m.m/fac. Arquitectura para la elaboración del Plan de Ordenamiento Territorial 
